\title{
Post-operative atrial fibrillation examined using whole-genome RNA sequencing in human left atrial tissue
}

\author{
Martin I Sigurdsson ${ }^{1 *}$, Louis Saddic ${ }^{1}$, Mahyar Heydarpour ${ }^{1}$, Tzuu-Wang Chang ${ }^{1}$, Prem Shekar ${ }^{2}$, Sary Aranki ${ }^{2}$, \\ Gregory S Couper ${ }^{2}$, Stanton K. Shernan ${ }^{1}$, Jochen D. Muehlschlegel ${ }^{1}$ and Simon C. Body ${ }^{1}$
}

\begin{abstract}
Background: Both ambulatory atrial fibrillation (AF) and post-operative AF (poAF) are associated with substantial morbidity and mortality. Analyzing the tissue-specific gene expression in the left atrium (LA) can identify novel genes associated with AF and further the understanding of the mechanism by which previously identified genetic variants associated with AF mediate their effects.

Methods: LA free wall samples were obtained intraoperatively immediately prior to mitral valve surgery in 62 Caucasian individuals. Gene expression was quantified on mRNA harvested from these samples using RNA sequencing. An expression quantitative trait loci (eQTL) analysis was performed, comparing gene expression between different genotypes of 1.0 million genetic markers, emphasizing genomic regions and genes associated with AF.

Results: Comparison of whole-genome expression between patients who later developed poAF and those who did not identified 23 differentially expressed genes. These included genes associated with the resting membrane potential modified by potassium currents, as well as genes within Wnt signaling and cyclic GMP metabolism. The eQTL analysis identified 16,139 cis eQTL relationships in the LA, including several involving genes and single nucleotide polymorphisms (SNPs) linked to AF. A previous relationship between rs3744029 and MYOZ1 expression was confirmed, and a novel relationship between rs6795970 and the expression of the SCN10A gene was identified.

Conclusions: The current study is the first analysis of the human LA expression landscape using high-throughput RNA sequencing. Several novel genes and variants likely involved in AF pathogenesis were identified, thus furthering the understanding of how variants associated with AF mediate their effects via altered gene expression.
\end{abstract}

Trial registration: ClinicalTrials.gov ID: NCT00833313, registered 5. January 2009

Keywords: Atrial fibrillation, Mitral valve surgery, eQTL, Expression quantitative trait loci

\section{Background}

The human left atrium (LA) has unique tissue and electrophysiological characteristics. It is associated with the pathogenesis of diseases in the conduction system, most notably atrial fibrillation (AF). To date, multiple genetic variants have been associated with $\mathrm{AF}$, including variants in the 4q25 locus containing the paired-like homeodomain 2 (PITX2) gene associated with the development of

\footnotetext{
* Correspondence: martiningi@gmail.com

'Department of Anesthesiology, Perioperative and Pain Medicine, Brigham and Women's Hospital/Harvard Medical School, 75 Francis Street, Boston, MA 02115, USA

Full list of author information is available at the end of the article
}

pulmonary vasculature [1], the 1q21 locus containing the member 3 potassium intermediate/small conductance calcium-activated channel member 3 (KCCN3) gene involved in cardiac conductance [2], and the 16q22 locus containing the zinc finger homeobox 3 (ZFHX3) gene with unknown functional significance [3]. Furthermore, variants within genes associated with ion conductance, such as genes encoding potassium and sodium channels, have been associated with AF [4-6]. Moreover, variants in 4q25 and 1q21 have also been associated with post-operative atrial fibrillation (poAF) after cardiac surgery $[7,8]$, indicating that the genetic background underlying both ambulatory $\mathrm{AF}$ and poAF may be similar. 
Previous studies examining the genetic variants associated with AF do not address their association with tissue-specific expression changes in the human LA that can contribute to the pathophysiology of AF. Comparing tissue-specific gene expression between sequence variants with expression quantitative loci (eQTL) analysis [9], can identify new variants associated with AF and further the understanding of how variants associated with AF mediate their effects.

Here, we used high throughput RNA sequencing to compare the gene expression pattern in the LA between those patients who did and did not develop poAF. Furthermore, we explored the genome-wide relationships between DNA sequence variants and whole-genome RNA expression of the LA, with a focus on regions and genes previously associated with AF and poAF.

\section{Methods}

\section{Patients}

Sixty three patients with self-reported two-generation Caucasian ancestry undergoing mitral valve repair or replacement between 12/2012 and 4/2014 were recruited for this study. We obtained LA tissue from the upper border of the routine left atriotomy incision in close proximity to the left pulmonary veins. The study was approved by the Institutional Review Board and all patients provided written informed consent.

Demographics were collected for all patients as well as data pertaining to the cardiac procedure. Images acquired from routine intraoperative transesophageal echocardiography were used to calculate LA volume normalized by body surface area using the ellipsoidal formula [10]. All patients were in sinus rhythm at the day of surgery. Patients were prospectively followed during the hospital stay for multiple clinical outcomes, including poAF. Similar to prior studies $[7,8]$, poAF was defined as any AF diagnosed by a clinician that developed during the primary hospitalization. Patients were monitored via continuous telemetry throughout their hospitalization.

\section{Sequencing and genotyping}

After harvesting, tissue samples were stored in RNAlater (Ambion; ThermoFisher Scientific) solution and whole RNA extraction was done by standardized methods. After reverse transcription of single-stranded RNA to double-stranded DNA, isolation of short fragments, and poly(A) addition and ligation of adaptors, 90 base-pair paired-end sequencing was performed on an Illumina HiSeq 2000 (Illumina, San Diego, CA). Prior to data analysis, adapter sequences were trimmed and low-quality reads were removed.

Using DNA isolated from whole blood, single nucleotide polymorphism (SNP) genotyping of 63 patients was performed using the Illumina Omni2.5 with exome content genotyping array (Illumina, San Diego, CA). One patient's DNA failed QC (genotyping call rate $<98 \%$ ) and was excluded from further analysis.

To test whether SNPs with eQTL association with differentially expressed genes in the LA of patients with poAF were associated with poAF in a larger cohort, we used a second cohort of 1,805 patients undergoing heart surgery at BWH $[7,8]$. DNA extracted from peripheral blood of study participants was used to genotype eight SNPs using the iPLEX Gold method on a Sequenom Mass Array system (Sequenom, San Diego, CA). Genotypes were called with the TypeAnalyzer Application.

\section{Alignment and Annotation}

Sequenced reads were aligned to the UCSC hg19 version of the human genome using TopHat2 (v2.0.9) [11] from the Bowtie2 algorithm [12]. Default parameters were used for the alignments except for mate inner distance and mate standard deviation, which were set at 165 and $37 \mathrm{bp}$, respectively. The aligned reads were sorted by chromosomal coordinates using Samtools [13] and then scored with HTseq-count [14] using the union mode for overlapping reads and the UCSC hg19 gene annotation file.

\section{Differential expression analysis}

All statistics and images were performed in $R$, version 3.0.1. [15]. A count matrix of all read counts was created from all sequenced samples. Differential expression and multivariate analysis was performed using DESeq2 [16]. This software was also used to create a matrix of normalized expression for each gene by calculating fragments per million mapped fragments (FPM). Utilizing the RNASeqPower package [17] in $\mathrm{R}$, we estimated that for alpha of 0.05 and power (1-beta) of 0.8 , with an estimated biological variation coefficient of 0.5 (humans), and around 30 cases of poAF, we could identify an expression difference of more than $50 \%$ for genes with more than 25 covering reads per gene, and an expression difference of more than $100 \%$ for genes with more than 10 covering reads per gene. Adjustment for multiple testing was done by calculating False Discovery Rate (FDR) using the method of Benjamini-Hochberg [18]. Genes with FDR-adjusted pvalue less than 0.1 and absolute $\log 2$ ratio fold change more than 0.5 were considered significant.

\section{Functional network analysis}

A left atrium co-expression network was generated by calculating the Pearson correlation coefficient between the normalized gene expression values for each gene in each patient. Genes with absolute correlation coefficient of more than 0.8 were considered to be co-expressed. The GeneMANIA server (www.genemania.org) was used to identify similarity between differentially expressed genes, predict 
their function, and list genes with similar functions [19]. The GeneMANIA algorithm was set to use the left atrium co-expression network in addition to its default colocalization, genetic interaction, pathway, physical interactions, shared protein domains, and predicted gene interaction networks under the automatic weighting method.

\section{eQTL analysis}

For data visualization and expression of quantitative trait loci (eQTL) analysis the normalized gene expression matrix was used. The eQTL analysis was done using the Matrix eQTL package in R [20]. For each SNP, this tests the association between the additive counts of the minor allele and the normalized expression of each gene. Results were focused on genes and SNPs in cis eQTL relationship, where the distance between the center of the gene and the SNP is less than 1 megabase. We considered $\mathrm{eQTL}$ associations with FDR adjusted $p$-value of less than 0.05 to be statistically significant.

The association between the eight SNPs chosen for genotyping in the cohort of 1,805 patients and poAF was analyzed using PLINK, v1.07 [21]. This was done with an additive genetic model after adjustment for age, gender, bypass time, and if aortic valve replacement was done. A $p$-value of less than 0.05 was considered statistically significant in this analysis.

\section{Results}

\section{Patient demographics}

In our study, 21 out of 62 (34\%) patients experienced post-op atrial fibrillation after mitral valve surgery. These patients were older and had a higher frequency of hypertension and statin usage (Table 1) compared to patients who did not experience poAF. Hypertension and statin usage were not significantly associated with poAF after correcting for age (data not shown). Although LA was substantially enlarged in both groups (maximum LA volume of $71 \mathrm{~mL} / \mathrm{m}^{2}$, compared to the normal range of 15 $41 \mathrm{~mL} / \mathrm{m}^{2}$ ), [22] there was no difference between patients with and without poAF. Similarly, left ventricular ejection fraction was similar between the two groups (Table 1).

Table 1 Patient demographics

\begin{tabular}{|c|c|c|c|c|}
\hline & All $(n=62)$ & No poAf $(n=41)$ & $\operatorname{poAF}(n=21)$ & $p$-value \\
\hline Female gender & 27 (44\%) & $17(41 \%)$ & $10(47 \%)$ & 0.85 \\
\hline Age (mean) & 58.7 & 56.0 & 64.8 & 0.006 \\
\hline \multicolumn{5}{|l|}{ Past Medical History } \\
\hline Smoking (Any) & $21(32 \%)$ & $12(29 \%)$ & $8(38 \%)$ & 0.68 \\
\hline Myocardial Infarction & $2(3 \%)$ & $1(2 \%)$ & $1(5 \%)$ & 1.00 \\
\hline Hypertension & $34(55 \%)$ & $18(44 \%)$ & 17 (81\%) & 0.01 \\
\hline Diabetes & $7(11 \%)$ & $4(11 \%)$ & $3(15 \%)$ & 0.94 \\
\hline Atrial fibrillation & $12(19 \%)$ & $7(17 \%)$ & $5(24 \%)$ & 0.76 \\
\hline Mitral Stenosis (severe) & $5(8 \%)$ & $3(8 \%)$ & $2(11 \%)$ & 0.65 \\
\hline \multicolumn{5}{|l|}{ Mitral Insufficiency } \\
\hline None & $1(2 \%)$ & $0(0 \%)$ & $1(5 \%)$ & \\
\hline Mild & $1(2 \%)$ & $1(2 \%)$ & $0(0 \%)$ & \\
\hline Moderate & $5(8 \%)$ & $4(10 \%)$ & $1(5 \%)$ & \\
\hline Severe & $55(89 \%)$ & $36(88 \%)$ & 19 (90\%) & 0.48 \\
\hline \multicolumn{5}{|l|}{ Preoperative medications } \\
\hline ACE inhibitor /ARB & $27(44 \%)$ & $16(39 \%)$ & $12(57 \%)$ & 0.28 \\
\hline$\beta$-blockers & $29(47 \%)$ & $18(44 \%)$ & $10(48 \%)$ & 0.99 \\
\hline Calcium channel inhibitors & $8(13 \%)$ & $7(17 \%)$ & $1(5 \%)$ & 0.38 \\
\hline HMG-CoA reductase inhibitor & 19 (31\%) & $9(21 \%)$ & $10(50 \%)$ & 0.05 \\
\hline \multicolumn{5}{|l|}{ Perioperative information } \\
\hline Left ventricular ejection fraction & 0.60 & 0.61 & 0.59 & 0.39 \\
\hline Left atrium volume $\left(\mathrm{ml} / \mathrm{m}^{2}\right)$ & 71 & 70 & 73 & 0.75 \\
\hline Cross-clamp time (min) & 106 & 108 & 101 & 0.58 \\
\hline Cardiopulmonary bypass time (min) & 144 & 146 & 143 & 0.85 \\
\hline
\end{tabular}

Patient characteristic of individuals included in the study. poAF - post-operative atrial fibrillation. ACE - angiotension-converting enzyme ARB - aldosterone II receptor blocker HGM-CoA -hydroxy-3-methylglutaryl-coenzyme 


\section{Expression Profile of the LA}

On average, RNA sequencing yielded 49.4 million (range 36.5-61.6 million) paired-end reads per sample and 96.1\% (range 93.9-96.9\%) of reads were aligned to the human genome by TopHat2. Of those 62.2\% (range 45.1-76.9\%) of the aligned reads were uniquely assigned to a single location. 21,878 genes had at least one read in at least one patient, with a large range of expression between genes. The mean normalized expression was 45.7 FPM (range 015,880; interquartile range 0.3-35.8 FPM). As expected, the most highly expressed genes were associated with core cardiac structural proteins, including cardiac actin-myosin chain (MYH6, ACTC1, MYL7) and other cardiac-specific genes including NPPA, MB, TTNT2, TTN and ANKRD1 (data not shown).

\section{Differential gene expression in patients with poAF}

We compared the gene expression in the LA between patients who did and did not develop poAF during their hospital stay (Fig. 1). After correcting for known and predetermined predictors of poAF (age, gender, cardiopulmonary bypass time, and LA volume) and accounting for multiple gene testing, we identified 23 differentially expressed genes (Table 2). Of note, we did not see expression changes in genes previously associated with AF by genome-wide association analysis, including PITX2 [1, 7], KCNN3 [2, 8], and ZFHX3 [3] (Fig. 2).

\section{Functional analysis of differentially expressed genes in poAF}

The list of differentially expressed genes in was submitted to the GeneMANIA algorithm [19] to identify functional connections. Overrepresentation analysis uncovered enrichment in two distinct functional classes (Fig. 3); the wingless integrated (wnt) signaling pathway (FDR adjusted $p$-value $=0.002)$ and the cyclic guanosine monophosphate metabolism pathway (FDR adjusted $p$-value $=0.002$ ).

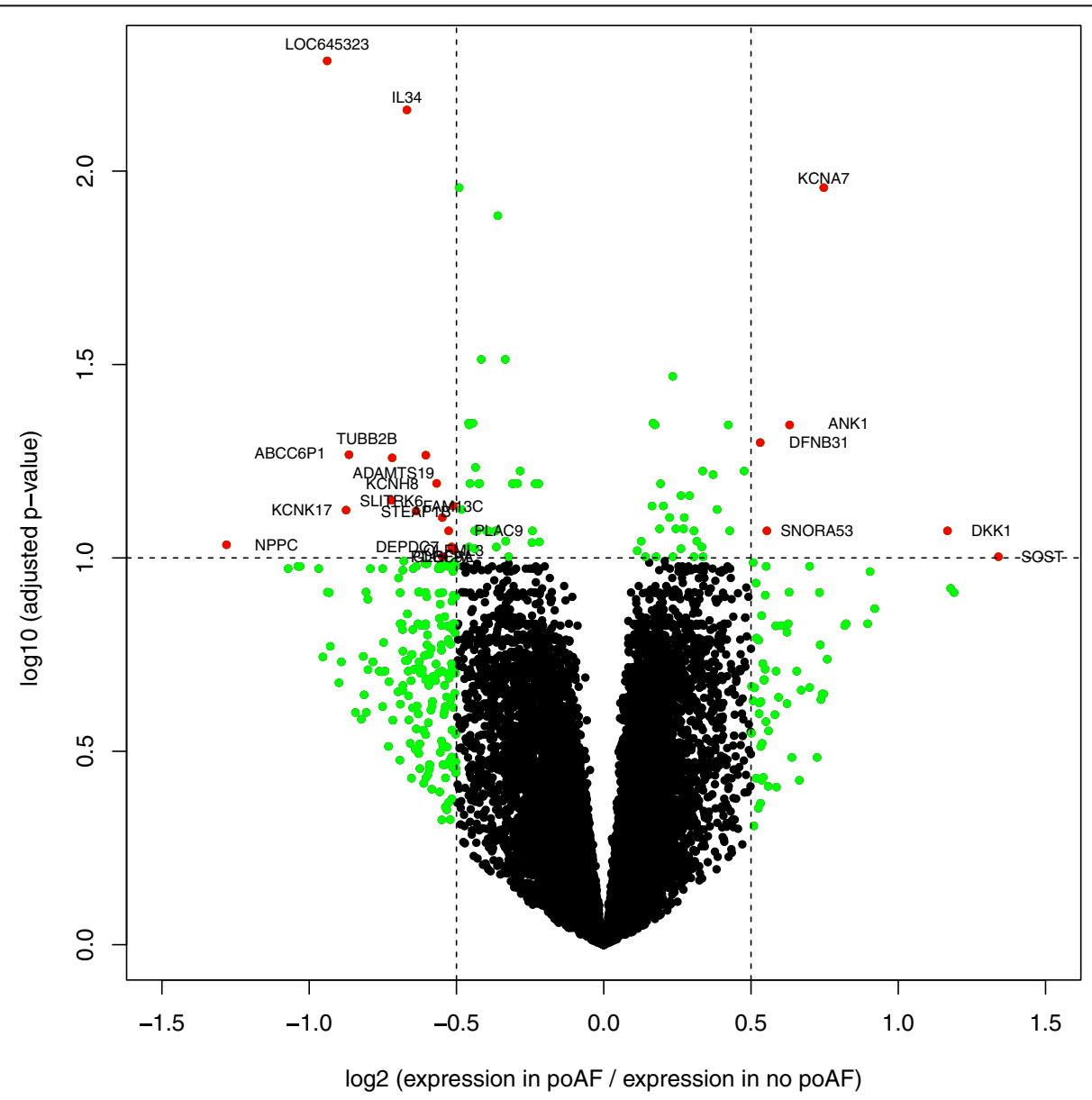

Fig. 1 Differentially expressed genes in patients with post-operative atrial fibrillation (poAF). A volcano plot comparing the expression of all genes in the human left atria between patients who had post-operative atrial fibrillation compared to those who did not. The $x$-axis shows the log 2 fold change and the $y$-axis shows the - $\log 10$ of the $p$-value adjusted for multiple testing. Dotted lines mark predetermined levels of significance; absolute log2 ratio $>0.5$ ( $x$-axis) or $p$-value adjusted for multiple testing $<0.1$ (y-axis). Green dots indicate genes that fulfill one significance criteria, red dost indicate genes that fulfill both 
Table 2 Genes with differential expression in the left atrium between patients with and without post-operative atrial fibrillation (poAF)

\begin{tabular}{|c|c|c|c|}
\hline Gene name & Fold change & $p$-value & FDR adj $p$-value \\
\hline LOC645323 & -0.9 & $3 \times 10^{-7}$ & 0.005 \\
\hline IL34 & -0.7 & $8 \times 10^{-7}$ & 0.007 \\
\hline KCNA7 & 0.7 & $3 \times 10^{-6}$ & 0.011 \\
\hline ANK1 & 0.6 & $4 \times 10^{-5}$ & 0.045 \\
\hline DFNB31 & 0.5 & $5 \times 10^{-5}$ & 0.050 \\
\hline$A B C C 6 P 1$ & -0.9 & $6 \times 10^{-5}$ & 0.054 \\
\hline TUBB2B & -0.6 & $6 \times 10^{-5}$ & 0.054 \\
\hline ADAMTS19 & -0.7 & $6 \times 10^{-5}$ & 0.055 \\
\hline KCNH8 & -0.6 & $1 \times 10^{-4}$ & 0.064 \\
\hline SLITRKG & -0.7 & $2 \times 10^{-4}$ & 0.071 \\
\hline FAM13C & -0.5 & $2 \times 10^{-4}$ & 0.073 \\
\hline KCNK17 & -0.9 & $2 \times 10^{-4}$ & 0.075 \\
\hline STEAPIB & -0.6 & $2 \times 10^{-4}$ & 0.076 \\
\hline RASGEFIC & -0.5 & $2 \times 10^{-4}$ & 0.079 \\
\hline DKK1 & 1.2 & $3 \times 10^{-4}$ & 0.085 \\
\hline PLAC9 & -0.5 & $3 \times 10^{-4}$ & 0.085 \\
\hline SNORA53 & 0.6 & $3 \times 10^{-4}$ & 0.085 \\
\hline NPPC & -1.3 & $4 \times 10^{-4}$ & 0.092 \\
\hline DEPDC7 & -0.5 & $4 \times 10^{-4}$ & 0.094 \\
\hline OLFML3 & -0.5 & $4 \times 10^{-4}$ & 0.096 \\
\hline CLEC9A & -0.5 & $5 \times 10^{-4}$ & 0.099 \\
\hline PDGFRL & -0.5 & $5 \times 10^{-4}$ & 0.099 \\
\hline SOST & 1.3 & $5 \times 10^{-4}$ & 0.099 \\
\hline
\end{tabular}

Genes were required to fulfill two criteria to be considered differentially expressed, the absolute of the log2 of the ratio of expression in patients with poAF and patients without poAF (fold change) had to be more than 0.5 , and the $p$-value for the expression difference adjusted to account for false discovery rate (FDR) was required to be less than 0.1

\section{Expression quantitative trait loci (eQTL) analysis of the left atrium}

In 62 patients, mean overall SNP genotyping rate for the $\sim 2.5$ million SNPs was $99.5 \%$. For analysis, we used the genotypes of all SNPs with at least $98 \%$ genotyping rate, a Hardy-Weinberg equilibrium $p$-value of $>10^{-5}$ and a minor allele frequency of $>10 \%$, yielding $1,028,887$ SNPs. A genome-wide cis eQTL analysis of the human LA was performed comparing the genotypes of all SNPs with the expression of all genes within 1 megabase.

At an FDR adjusted $p$-value $<0.05$, a total of 16,139 cis eQTL relationships between 13,927 different SNPs and 1,880 different genes were found (Fig. 4, Additional file 1). After exclusion of poorly expressed gene-SNP pairs $(<0.1$ FPM) and eQTL relationships including the Y chromosome, the strongest cis eQTL association was between rs78042921 and the expression of the X-Ray Radiation Resistance Associated 1 (XRRA1) gene on chromosome 11, along with other SNPs in high linkage disequilibrium with rs78042921. The highest number of eQTL relationships was found between SNPs and the ring finger protein 5, E3 ubiquitin protein ligase pseudogene 1 (RNF5P1) on chromosome $8(n=748)$. A cluster of genes on chromosome 6 encoding members of the major histocompatibility complex $(\mathrm{MHC})$ class I including HLA-DRB5, ZFP57, MICA, MICB, ZNRD1-AS1, CDSN, $H L A-D R B 6, P S O R S 1 C 2$ and $H L A-H$ were also among the genes with the highest number of cis eQTLs.

\section{eQTL analysis of variants associated with AF by genome-wide association studies}

The LA eQTL results were analyzed to identify significant cis eQTL relationships that included regions within $500 \mathrm{~kb}$ of the location of SNPs previously shown to be associated with AF in ambulatory and surgical cohorts [23]. This uncovered 9 such relationships (Table 3), including a strong eQTL association between rs3740293 and MYOZ1

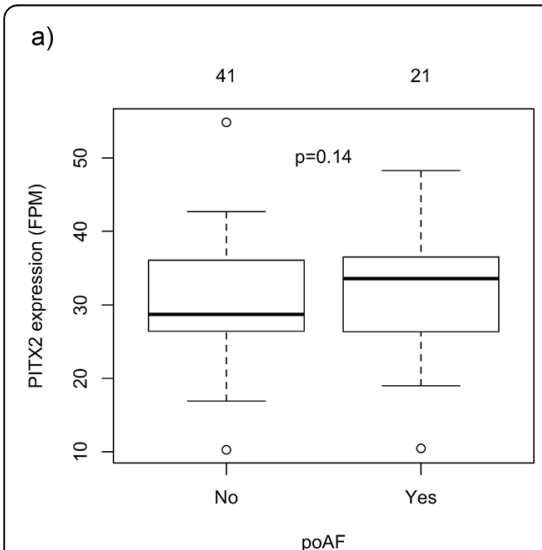

b)

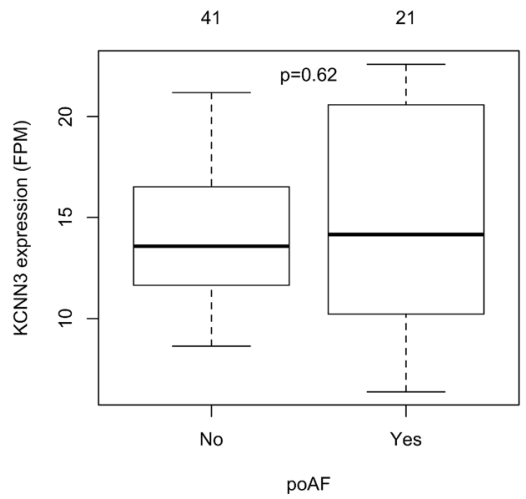

c)

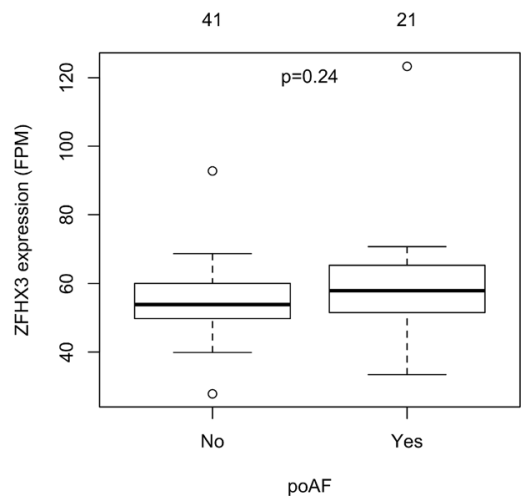

Fig. 2 Expression of PITX2, KCNN3, and ZFHX3 in the LA of patients with and without post-operative atrial fibrillation (poAF). Boxplots of the normalized expression of a PITX2, b KCNN3 and $\mathbf{c}$ ZFHX3 between patients with and without poAF. The number above each box shows the number of patients in each group. FPM - Fraction per million mapped fragments 


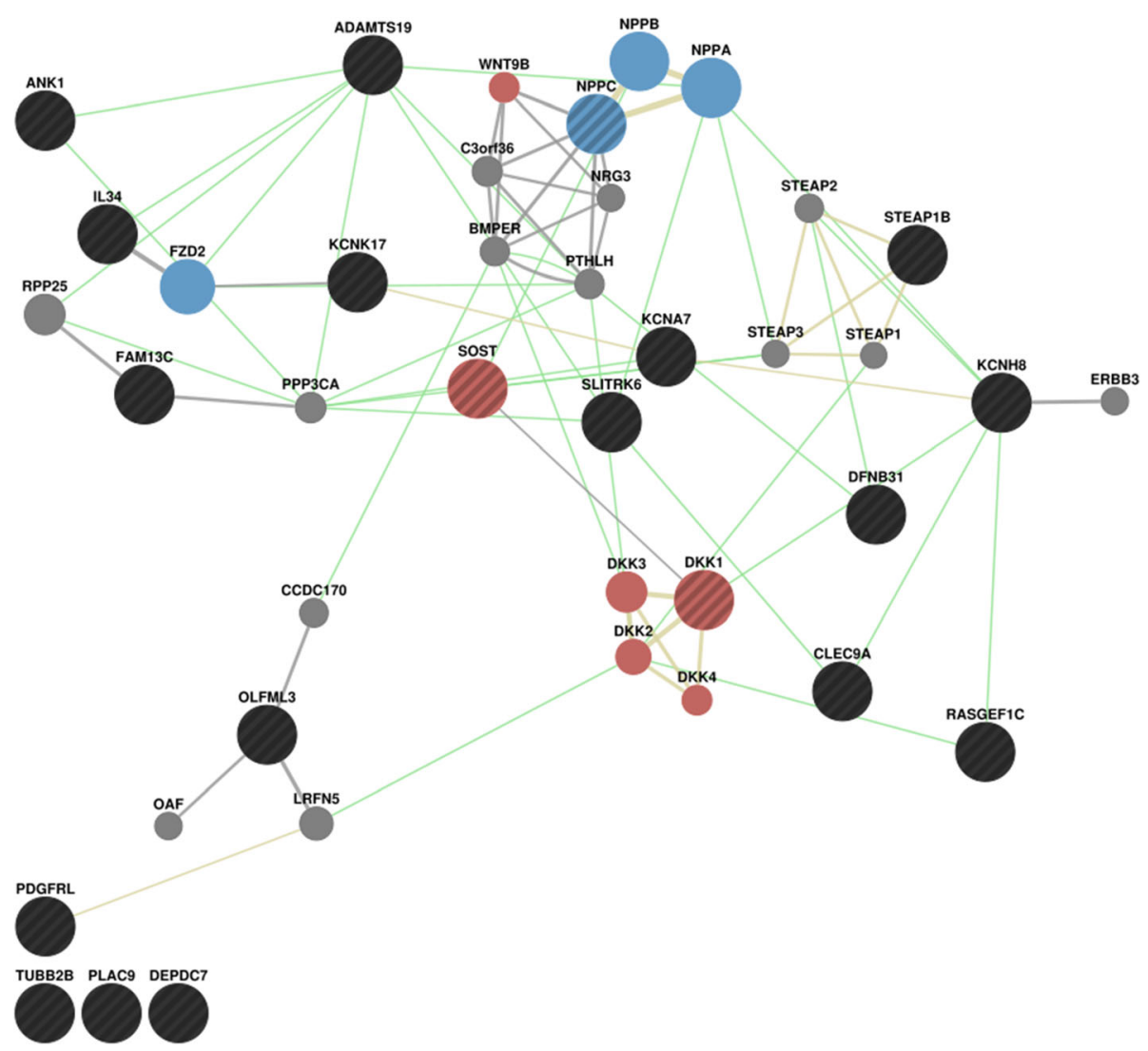

Fig. 3 Gene Network Analysis. Result of the functional network analysis of the list of genes with differential expression in poAF by the GeneMANIA algorithm [19]. Connections were assessed using the list of differentially expressed genes (black, black stripes) in the context of a left atrial co-expression network (gray connections) and the default GeneMANIA networks on genetic interactions (green) or shared protein domains (yellow). Two gene function categories were overrepresented in the output, the Wnt Signaling pathway (red) and the cGMP metabolic process (blue)

expression (Fig. 5a, Additional file 1). The genotype of rs3740293 has previously been associated with the expression of the MYOZ1 gene in left and right atrial tissue [24], and this SNP is in high linkage disequilibrium (LD) with rs10824026, which has also been associated with AF [24, 25].
eQTL analysis of variants associated with AF by candidate-gene analysis

The LA eQTL results were analyzed to identify significant cis eQTL relationships involving genes previously shown to be associated with AF via candidate gene analysis $[4,6]$.

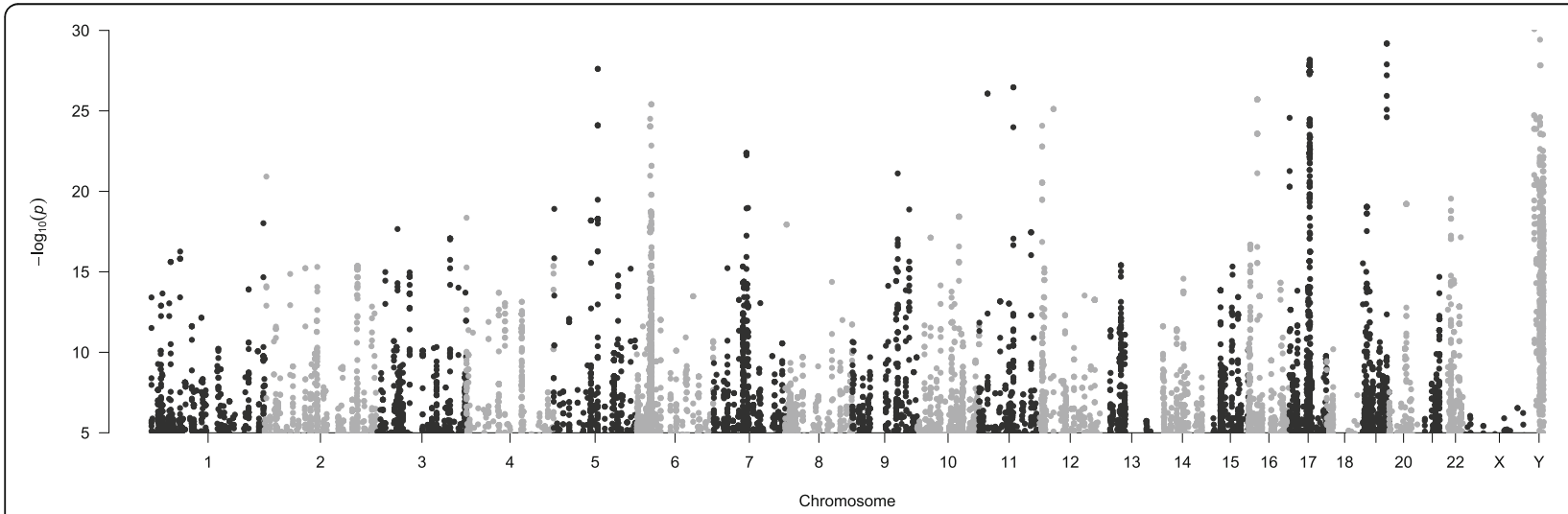

Fig. 4 Genomic location of cis-eQTL relationships in the human LA. A Manhattan plot of the genomic location of all significant cis-eQTL (at false discovery adjusted $p<0.05$ ) relationships in the human left atrium 
Table 3 cis eQTL relationships involving variants close to regions associated with AF via genome-wide association studies

\begin{tabular}{llllll}
\hline SNP & Gene & Chromosome & SNP Location & $p$-value & $\begin{array}{c}\text { FDR adjusted } \\
p \text {-value }\end{array}$ \\
\hline rs60632610 & MYOZ1 & 10 & $75,415,677$ & $1.7 \times 10^{-14}$ & $3.5 \times 10^{-10}$ \\
rs10894154 & CNTN5 & 11 & $99,781,954$ & $2.8 \times 10^{-6}$ & 0.007 \\
rs4437927 & FMO6P & 1 & $170,196,342$ & $6.2 \times 10^{-6}$ & 0.01 \\
rs9409797 & MIR27B & 9 & $97,276,713$ & $6.5 \times 10^{-6}$ & 0.01 \\
rs6590399 & CNTN5 & 11 & $99,759,525$ & $7.4 \times 10^{-6}$ & 0.01 \\
rs8847 & RIT1 & 1 & $155,259,323$ & $1.0 \times 10^{-5}$ & 0.02 \\
rs305043 & PAQR5 & 15 & $70,088,292$ & $1.8 \times 10^{-5}$ & 0.03 \\
rs10908463 & RIT1 & 1 & $155,286,258$ & $2.2 \times 10^{-5}$ & 0.03 \\
rs11264341 & GBAP1 & 1 & $155,151,493$ & $3.1 \times 10^{-5}$ & 0.04 \\
\hline
\end{tabular}

Shown are all significant cis eQTL relationships (at FDR-adjusted $p$-value $<0.05$ ) between SNPs previously characterized as being associated with AF and genes within $500 \mathrm{~kb}$ [23]. For each gene, the most significant eQTL relationship is shown. Variant location is in coordinates from the UCSC hg19 genome annotation

This analysis identified ten eQTL relationships involving the SCN10A gene and adjacent SNPs in high LD (Additional file 1). These included rs6800541, previously associated with AF, and rs6795970, a missense variant in the SNC10A gene (Fig. 5b).

\section{eQTL analysis of variants associated genes with} differential expression in the LA of patients with poAF

The LA eQTL results were analyzed for significant cis eQTLs including those genes that were differentially expressed between those patients that did and did not develop poAF in the LA (Table 2). This analysis identified cis eQTL relationships involving four out of the 23 differentially expressed genes (Table 4). These SNPs could potentially be novel candidate markers involved in AF or poAF.

\section{Association of novel SNPs with poAF in a larger cohort} We tested whether variants with eQTL relationships with differentially expressed genes in the LA of our surgical patients were also associated with poAF in a larger cohort of patients undergoing cardiac surgery (Table 5). This was accomplished by genotyping four SNPs with such associations in an additional cohort of 1,805 Caucasian individuals undergoing heart surgery (Table 5). One out of four SNPs, namely rs10759721, was found to be associated with poAF in this larger cohort. This SNP has an eQTL

\section{a)}

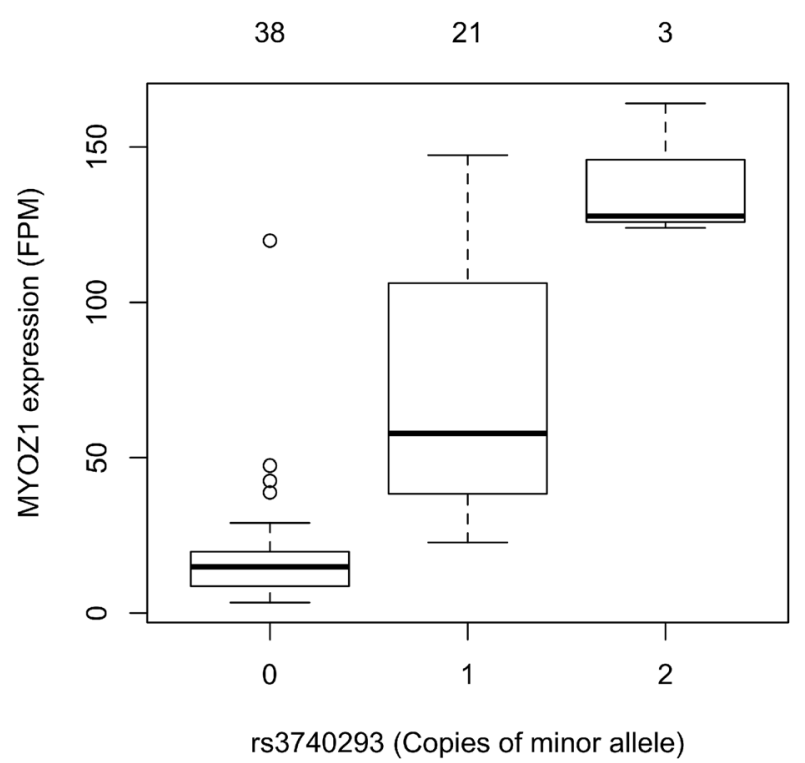

b)

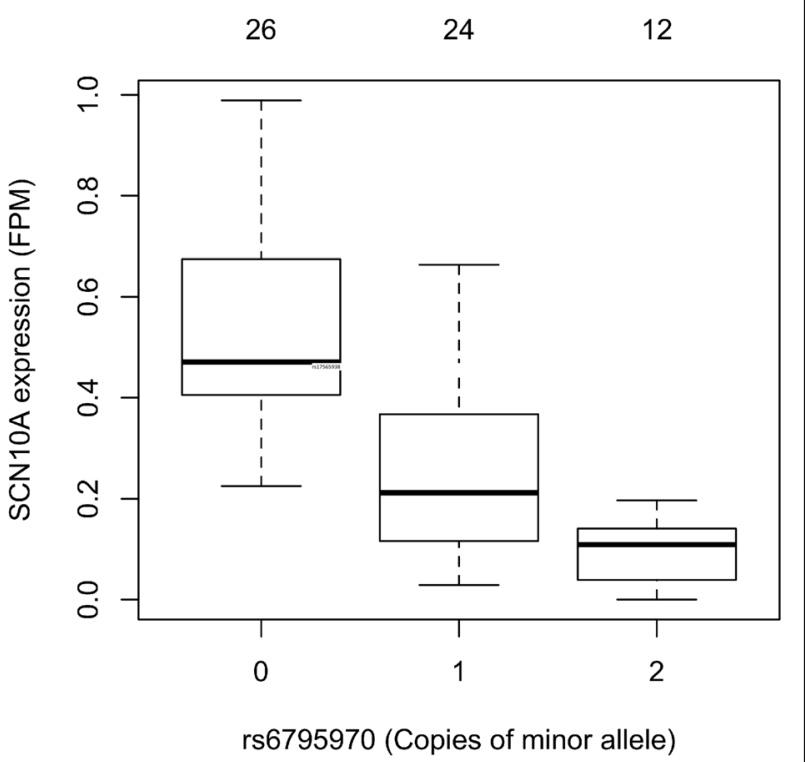

Fig. 5 cis eQTL relationships of SNPs and genes associated with AF. Boxplots of the normalized expression of each gene (y-axis) and the genotype (allele counts of minor allele) for a MYOZ1 and rs3740293 and b SCN10A gene and rs6795970. The number above each box shows the number of patients with each genotype. FPM - Fraction fragments per million mapped fragments 
Table 4 cis eQTL relationships of genes differentially expressed in poAF

\begin{tabular}{llllll}
\hline SNP & Gene & Chromosome & Location & $p$-value & FDR adjusted $p$-value \\
\hline rs10759721 & DFNB31 & 9 & $117,326,038$ & $3.7 \times 10^{-7}$ & 0.001 \\
rs4802546 & KCNA7 & 19 & $49,570,686$ & $9.4 \times 10^{-6}$ & 0.02 \\
rs4911071 & SLITRK6 & 13 & $85,911,610$ & $1.6 \times 10^{-5}$ & 0.03 \\
rs2393581 & FAM13C & 10 & $61,801,282$ & $3.3 \times 10^{-5}$ & 0.04 \\
\hline
\end{tabular}

Significant cis eQTL relationships (at FDR-adjusted $p$-value $<0.05$ ) between genomic variants and genes with differential expression in our poAF study (Table 2).

For each gene, the most significant eQTL relationship is shown. Variant location is in hg19 coordinates

relationship with the expression of the DFNB31 gene. The $D F N B 31$ gene was more highly expressed in the LA of patients with poAF, and the eQTL analysis identified a negative correlation between the expression of the DFNB31 gene and the number of minor alleles $(\mathrm{T})$ of rs10759721. Therefore the minor allele may be associated with protection against poAF. This relationship matches the results from the larger cohort, where individuals with the minor allele had reduced odds of poAF (OR 0.81 per minor allele (T), 95\% CI 0.69-0.95, $p=0.01$ ).

\section{Discussion}

This study analyzed human LA gene expression differences and eQTL relationships in the human LA, emphasizing expression differences and eQTL relationship affecting atrial fibrillation. Several authors have studied the gene expression profile of the human LA in the context of comparing it to the profile of the right atrium, and also comparing right atrial or right atrial appendage expression profiles of patients with and without poAF [24, 26-28]. Nevertheless, the limitations of these other studies include small patient numbers, the use of the left or right atrial appendage as a surrogate for LA tissue, and the inherent complications of older technological approaches such as microarrays for expression profiling.

The most significant differentially expressed gene identified, LOC645323, is a long noncoding RNA known to be highly expressed in neuronal tissue. Transgenic knockout mice of this gene resulted in no identifiable phenotype, and no other studies describing its function in humans have been reported [29]. This gene however resides in a highly conserved region that includes the miRNA miR-92,

Table 5 Association of candidate SNPs with poAF in a large independent cohort of surgical patients

\begin{tabular}{lllll}
\hline SNP & Associated Gene & OR & $95 \%$ Cl & $p$-value \\
\hline rs10759721 & DFNB31 & 0.81 & $0.69-0.95$ & 0.0100 \\
rs10817638 & DFNB31 & 0.84 & $0.71-0.99$ & 0.0415 \\
rs60632610 & MYOZ1 & 1.12 & $0.90-1.39$ & 0.31 \\
rs4802546 & KCNA7 & 1.00 & $0.78-1.29$ & 1.00
\end{tabular}

4 SNPs identified as having an association with poAF in the eQTL study were validated in an independent cohort of 1805 surgical patients. Shown is the odds ratio, $95 \% \mathrm{Cl}$, and unadjusted $\mathrm{p}$-value for the association, after adjustment for age, gender, bypass time and the performance of valve surgery and myocyte enhancer factor 2c (MEF2C) gene, a myocardial transcription factor whose protein levels are associated with arrhythmias in mice [30]. Long noncoding RNA molecules affect both local and distal gene transcription, so it is possible that the expression of LOC645323 influences the expression of the adjacent the $M E F 2 C$ gene, resulting in the observed poAF phenotype.

From the list of genes with differential expression between patients with and without poAF there were several involving potassium channels (Table 3 ). In addition to prior association of potassium channel genes (KCNN3, KCNA5 and $K C N E 1-5)$ with AF described by others [4], we identified three additional genes in the potassium channel gene family with differential expression in poAF. The KCNA7 gene encodes a voltage-gated potassium channel [31] the KCNH8 gene encodes a voltage-dependent potassium channel [32], and the KCNK17 gene codes for a potassium channel that contributes to the resting membrane potential [33]. None of these genes has thus far not been associated with an arrhythmia. Interestingly, decreased left atrial expression of the PITX2 gene in mice was found to be associated with lower resting membrane potential via modification of background potassium currents [34]. Furthermore, a lower resting membrane potential was associated with increased sensitivity to sodium channel blockers to prevent $\mathrm{AF}$ in the experimental animals. These findings and ours suggest that there might be a subset of patients with AF whose arrhythmia is caused by altered resting membrane potential mediated by potassium currents.

Similar to a recent study [35], we did not observe differences in the PITX2 expression between subjects with and without poAF. This indicates that the identified association between DNA variants within these genes and AF are not mediated via overall expression changes, although it is possible that this also reflects differences between the study population of patients with mitral valve disease and ambulatory populations with $\mathrm{AF}$ in the absence of mitral valve disease.

Network analysis identified that the list of differentially expressed genes was enriched in two functional pathways, namely Wnt signaling and cGMP metabolic process. Wnt proteins are intracellular signaling molecules that trigger distinct canonical and noncanonical pathways. In the 
canonical pathway, binding of Wnt proteins at the plasma membrane triggers a phosphorylation cascade that stabilizes $\beta$-catenin, a nuclear co-activator of several transcription factors [36]. This pathway plays a significant role in early cardiac development, where it regulates the generation of mesoderm formation and cell adhesion in cardiomyocytes [37]. It has been suggested that reactivation of Wnt signaling in the aging heart contributes to cardiac fibrosis, which may contribute to a further risk of AF [38]. More recently, analysis of Pitx2 loss-of function mouse models revealed that complex relationships exist between the expression of the Pitx2 gene and calcium homeostasis via Wnt signaling [39]. The authors believe that this mechanism could be responsible for the association between the PITX2 gene and AF. The results of our study further support involvement of the Wnt signaling pathway in the pathogenesis of AF in humans, potentially through cardiac fibrosis and its effect on calcium current homeostasis.

The cGMP pathway is involved in several signaling pathways including nitric oxide (NO) mediated cardiomyocyte ion-channel function. Indeed, platelet cGMP levels, used as a surrogate assessment for the activity of the NO pathway, were found to be reduced in patients with AF compared to patients in sinus rhythm [40]. In addition to its role in regulating vascular tone, $\mathrm{NO}$ also effects cardiac ionic currents, including potassium currents regulating the resting membrane potential, and thus may play a role in the pathogenesis of AF [41].

Several of the cis eQTL relationships identified, involve the major histocompatibility complex (MHC). Furthermore, the $U T Y$ gene, the strongest eQTL relationship, is also a minor histocompatibility complex gene. These findings are similar to several eQTL studies from other tissues that identify MHC as a major eQTL source [42]. A cautious interpretation of eQTLs including the MHC regions has been encouraged, as the extreme variability of this region can bias expression quantification of microarray and sequencing methods towards detecting the reference genome sequence. This can render individuals with many minor alleles of MHC genes less likely to have detectable expression of the MHC genes, creating spurious eQTL relationships [42].

Among the strongest cis eQTL relationships involving regions associated with ambulatory AF were relationships between variants on 10q22 including rs3740293 and the MYOZ1 gene (Table 3, Fig. 5). Lin et al. also identified a cis eQTL relationship between rs3740293 and the expression of MYOZ1 with microarray and quantitative real-time polymerase chain reaction [24]. Previously, the SNP rs10824026 in this locus has been strongly associated with AF [25]. This SNP is in high LD with many of the SNPs we identified to be associated with MYOZ1 expression, including rs3740293. The
MYOZ1 gene is expressed in heart and skeletal muscle and is involved in calcineurin signaling which establishes a stable sarcomere. Based on our eQTL findings, it is possible that the identified relationship between rs3740293 and ambulatory AF, stem from altered expression of this gene in minor allele carriers, resulting in altered sarcomere mechanics and calcium homeostasis contributing to poAF [43].

Several cis eQTL relationships were found involving the expression of the SCN1OA gene. The SCN1OA gene encodes a voltage-gated sodium channel $\mathrm{Na}_{\mathrm{v}} 1.8$ [44], and variants in this gene have been associated both with variations in the $\mathrm{PR}$ interval and with $\mathrm{AF}$ [45]. The rs6795970 SNP leads to a missense mutation in SCN10A and is in a high LD with rs6800541, which resides in one of its introns. Recently, rs6795970 was found to be protective against $\mathrm{AF}$ in a lone AF cohort [6]. Functional studies with the rs6795970 variant in cell culture have revealed increased peak and sustained currents, and slowing of fast inactivation of the cell membrane [6]. Our results indicate that the membrane electrophysiological properties associated with the SNP may be mediated by decreased expression of the SCN10A gene or the expression of the SCN10A gene is decreased as a response to this gain-of function mutation.

Several limitations of the study should be mentioned. Any duration of arrhythmia was included in in our definition of poAF. This was done under the understanding that although poAF of short duration might not have a clinical relevance it likely indicates a genetic predisposition towards the arrhythmia. We sampled tissue from the LA free wall, which is structurally and functionally more similar to the tissue involved in the pathophysiology of AF. This however limited the number of patients included, and the amount of available tissue. The small amount of tissue available precluded us from performing independent biological replicates to minimize variability in RNA expression estimates. Furthermore, the study cohort of patients presenting for mitral valve surgery limits the generalizability of the results to other populations. In particular, the larger cohort used to test the association of SNPs in eQTL relationship with differentially expressed genes stems from a surgical cohort undergoing aortic valve and coronary bypass surgery. Finally, the expression changes observed in poAF might not translate completely to ambulatory AF, as they represent two different processes. We do however think that some of the genetic predisposition towards poAF is shared with ambulatory AF. This is supported by the ability of our group to correlate genetic variants associated with ambulatory AF to poAF after cardiac surgery using the same definition of poAF of any duration [7]. 


\section{Conclusion}

In summary, we describe the expression landscape of the human LA with a focus on those genes involved in poAF. It is likely that either poAF or ambulatory AF represent a final common phenotype of alteration in multiple different electrophysiological pathways. We found association between poAF and both the resting membrane potential as well as pathways associated with calcium homeostasis and atrial fibrosis. The results of the eQTL analysis further the understanding of how variants associated with AF and poAF mediate their effects, in particular how variants adjacent to the MYOZ1 and SCN10A genes might modulate their effects on AF risk. The genetic signatures of poAF and ambulatory AF have thus far proven to be highly similar despite different environmental insults associated with the onset of the phenotype. Therefore, it is our hope that our results can further both the understanding of AF pathophysiology in both post-surgical and ambulatory populations.

\section{Additional file}

Additional file 1: All cis eQTL relationships in $L A$ at false discovery rate (FDR)-adjusted $p$-value $<0.05$. Location is in hg19 coordinates. Mean gene expression is in normalized FPM (fragments per million mapped fragments). (TXT $2516 \mathrm{~kb}$ )

\section{Abbreviations}

AF: Atrial fibrillation; EQTL: Expression quantitative trait loci; FDR: False discovery rate; LA: Left atrium; PoAF: Post-operative atrial fibrillation; SNP: Single nucleotide polymorphism

\section{Acknowledgements}

We acknowledge the contribution to sample collection by the perioperative genomics center and its staff: James Gosnell, RN; Kujtim Bodinaku, MD; Svetlana Gorbatov, MPH.

\section{Funding}

Grants from the National Heart, Lung, and Blood Institute (R01HL098601), (1R01HL114823, SCB).

\section{Availability of data and materials}

Data from the LA datasets analysed during the current study available from the corresponding author upon reasonable request.

\section{Authors' contributions \\ MIS: study design, data analysis, manuscript writing and revision. LS: data analysis, critical revision of manuscript, $\mathrm{MH}$ : data analysis, critical revision of manuscript, TWX: data analysis, critical revision of manuscript, PS: obtained tissue from patients, critical revision of manuscript, SA: obtained tissue from patients, critical revision of manuscript, GSC: obtained tissue from patients, critical revision of manuscript, SKS: study conception, critical revision of the manuscript, JDM: study design and conception, data analysis, critical revision of manuscript, SCB: study design and conception, data analysis, critical revision of manuscript. All authors have reviewed and approve of the final version of the manuscript.}

\section{Competing interests}

The authors declare that they have no competing interests

\section{Consent for publication}

Not applicable

\section{Ethics approval and consent to participate}

The study was approved by the Institutional Review Board at Brigham and Women's Hospital and all patients provided written informed consent.

\section{Publisher's Note}

Springer Nature remains neutral with regard to jurisdictional claims in published maps and institutional affiliations.

\section{Author details}

${ }^{1}$ Department of Anesthesiology, Perioperative and Pain Medicine, Brigham and Women's Hospital/Harvard Medical School, 75 Francis Street, Boston, MA 02115, USA. ²Division of Cardiac Surgery, Department of Surgery, Brigham and Women's Hospital/Harvard Medical School, Boston, MA, USA.

Received: 2 August 2016 Accepted: 25 April 2017

Published online: 02 May 2017

\section{References}

1. Gudbjartsson DF, Arnar DO, Helgadottir A, Gretarsdottir S, Holm H, Sigurdsson A, Jonasdottir A, Baker A, Thorleifsson G, Kristjansson K, et al. Variants conferring risk of atrial fibrillation on chromosome $4 q 25$. Nature. 2007:448(7151):353-7.

2. Ellinor PT, Lunetta KL, Glazer NL, Pfeufer A, Alonso A, Chung MK, Sinner MF, de Bakker PI, Mueller M, Lubitz SA, et al. Common variants in KCNN3 are associated with lone atrial fibrillation. Nat Genet. 2010;42(3):240-4.

3. Benjamin EJ, Rice KM, Arking DE, Pfeufer A, van Noord C, Smith AV, Schnabel RB, Bis JC, Boerwinkle E, Sinner MF, et al. Variants in ZFHX3 are associated with atrial fibrillation in individuals of European ancestry. Nat Genet. 2009;41(8):879-81.

4. Olesen MS, Nielsen MW, Haunso S, Svendsen JH. Atrial fibrillation: the role of common and rare genetic variants. Eur J Hum Genet. 2014;22(3):297-306.

5. Sinner MF, Ellinor PT, Meitinger T, Benjamin EJ, Kaab S. Genome-wide association studies of atrial fibrillation: past, present, and future. Cardiovasc Res. 2011:89(4):701-9.

6. Jabbari J, Olesen MS, Yuan L, Nielsen JB, Liang B, Macri V, Christophersen IE, Nielsen N, Sajadieh A, Ellinor PT, et al. Common and rare variants in SCN10A modulate the risk of atrial fibrillation. Circ Cardiovasc Genet. 2015;8(1):64-73.

7. Body SC, Collard CD, Shernan SK, Fox AA, Liu KY, Ritchie MD, Perry TE, Muehlschlegel JD, Aranki S, Donahue BS, et al. Variation in the $4 q 25$ chromosomal locus predicts atrial fibrillation after coronary artery bypass graft surgery. Circ Cardiovasc Genet. 2009;2(5):499-506.

8. Sigurdsson MI, Muehlschlegel JD, Fox AA, Heydarpour M, Lichtner P, Meitinger T, Collard CD, Shernan SK, Body SC. Genetic variants associated with atrial fibrillation and PR interval following cardiac surgery. J Cardiothorac Vasc Anesth. 2015;29(3):605-10.

9. Cookson W, Liang L, Abecasis G, Moffatt M, Lathrop M. Mapping complex disease traits with global gene expression. Nat Rev Genet. 2009;10(3):184-94.

10. Khankirawatana B, Khankirawatana S, Porter T. How should left atrial size be reported? Comparative assessment with use of multiple echocardiographic methods. Am Heart J. 2004;147(2):369-74.

11. Kim D, Pertea G, Trapnell C, Pimentel H, Kelley R, Salzberg SL. TopHat2: accurate alignment of transcriptomes in the presence of insertions, deletions and gene fusions. Genome Biol. 2013;14(4):R36.

12. Langmead B, Trapnell C, Pop M, Salzberg SL. Ultrafast and memory-efficient alignment of short DNA sequences to the human genome. Genome Biol. 2009;10(3):R25.

13. Li H, Handsaker B, Wysoker A, Fennell T, Ruan J, Homer N, Marth G, Abecasis G, Durbin R. Genome project data processing S: the sequence alignment/ map format and SAMtools. Bioinformatics. 2009;25(16):2078-9.

14. Anders S, Pyl PT, Huber W. HTSeq-a Python framework to work with highthroughput sequencing data. Bioinformatics. 2015;31(2):166-9.

15. R Development Core Team. R: A language and environment for statistical computing. Vienna: R Foundation for Statistical Computing; 2011.

16. Love MI, Huber W, Anders S. Moderated estimation of fold change and dispersion for RNA-Seq data with DESeq2. Genome Biol. 2014;15(12):550.

17. Hart SN, Therneau TM, Zhang Y, Poland GA, Kocher JP. Calculating sample size estimates for RNA sequencing data. J Comput Biol. 2013;20(12):970-8.

18. Benjamini $Y$, Hochberg $Y$. Controlling the false discovery rate: a practical and powerful aproach to multiple testing. J R Stat Soc B. 1995;57(1):289-300.

19. Warde-Farley D, Donaldson SL, Comes O, Zuberi K, Badrawi R, Chao P, Franz M, Grouios C, Kazi F, Lopes CT, et al. The GeneMANIA prediction server: 
biological network integration for gene prioritization and predicting gene function. Nucleic Acids Res. 2010;38(Web Server issue):W214-220.

20. Shabalin AA. Matrix eQTL: ultra fast eQTL analysis via large matrix operations. Bioinformatics. 2012;28(10):1353-8.

21. Purcell S, Neale B, Todd-Brown K, Thomas L, Ferreira MA, Bender D, Maller J, Sklar P, de Bakker PI, Daly MJ, et al. PLINK: a tool set for whole-genome association and population-based linkage analyses. Am J Hum Genet. 2007;81(3):559-75.

22. Aune E, Baekkevar M, Roislien J, Rodevand O, Otterstad JE. Normal reference ranges for left and right atrial volume indexes and ejection fractions obtained with real-time three-dimensional echocardiography. Eur J Echocardiogr. 2009;10(6):738-44.

23. Beck T, Hastings RK, Gollapudi S, Free RC, Brookes AJ. GWAS central: a comprehensive resource for the comparison and interrogation of genomewide association studies. Eur J Hum Genet. 2014;22(7):949-52.

24. Lin H, Dolmatova EV, Morley MP, Lunetta KL, McManus DD, Magnani JW, Margulies KB, Hakonarson H, del Monte F, Benjamin EJ, et al. Gene expression and genetic variation in human atria. Heart Rhythm. 2014;11(2):266-71.

25. Ellinor PT, Lunetta KL, Albert CM, Glazer NL, Ritchie MD, Smith AV, Arking DE, Muller-Nurasyid M, Krijthe BP, Lubitz SA, et al. Meta-analysis identifies six new susceptibility loci for atrial fibrillation. Nat Genet. 2012;44(6):670-5.

26. Wang J, Wang Y, Han J, Li Y, Xie C, Xie L, Shi J, Zhang J, Yang B, Chen D, et al. Integrated analysis of microRNA and mRNA expression profiles in the left atrium of patients with nonvalvular paroxysmal atrial fibrillation: Role of miR-146b-5p in atrial fibrosis. Heart Rhythm. 2015;12(5):1018-26.

27. Liu H, Qin H, Chen GX, Liang MY, Rong J, Yao JP, Wu ZK. Comparative expression profiles of microRNA in left and right atrial appendages from patients with rheumatic mitral valve disease exhibiting sinus rhythm or atrial fibrillation. J Transl Med. 2014;12:90.

28. Deshmukh A, Barnard J, Sun H, Newton D, Castel L, Pettersson G, Johnston D, Roselli E, Gillinov AM, McCurry K, et al. Left atrial transcriptional changes associated with atrial fibrillation susceptibility and persistence. Circ Arrhythm Electrophysiol. 2015;8(1):32-41.

29. Oliver PL, Chodroff RA, Gosal A, Edwards B, Cheung AF, Gomez-Rodriguez J, Elliot G, Garrett L, Lickiss T, Szele F, et al. Disruption of Visc-2, a brainexpressed conserved long noncoding RNA, does not elicit an overt anatomical or behavioral phenotype. Cereb Cortex. 2014;25:3572.

30. Hattori K, Nakamura K, Hisatomi Y, Matsumoto S, Suzuki M, Harvey RP, Kurihara H, Hattori S, Yamamoto T, Michalak M, et al. Arrhythmia induced by spatiotemporal overexpression of calreticulin in the heart. Mol Genet Metab. 2007;91(3):285-93.

31. Bardien-Kruger S, Wulff H, Arieff Z, Brink P, Chandy KG, Corfield V. Characterisation of the human voltage-gated potassium channel gene, KCNA7, a candidate gene for inherited cardiac disorders, and its exclusion as cause of progressive familial heart block I (PFHBI). Eur J Hum Genet. 2002;10(1):36-43.

32. Zou A, Lin Z, Humble M, Creech CD, Wagoner PK, Krafte D, Jegla TJ, Wickenden AD. Distribution and functional properties of human KCNH8 (Elk1) potassium channels. Am J Physiol Cell Physiol. 2003;285(6):C1356-1366.

33. Decher N, Maier M, Dittrich W, Gassenhuber J, Bruggemann A, Busch AE, Steinmeyer K. Characterization of TASK-4, a novel member of the pH-sensitive, two-pore domain potassium channel family. FEBS Lett. 2001;492(1-2):84-9.

34. Syeda F, Holmes AP, Yu TY, Tull S, Kuhlmann SM, Pavlovic D, Betney D, Riley G, Kucera JP, Jousset F, et al. PITX2 modulates atrial membrane potential and the antiarrhythmic effects of sodium-channel blockers. J Am Coll Cardiol. 2016;68(17):1881-94.

35. Gore-Panter SR, Hsu J, Hanna P, Gillinov AM, Pettersson G, Newton DW, Moravec CS, Van Wagoner DR, Chung MK, Barnard J, et al. Atrial Fibrillation associated chromosome 4q25 variants are not associated with PITX2C expression in human adult left atrial appendages. PLoS One. 2014;9(1):e86245.

36. Rao TP, Kuhl M. An updated overview on Wnt signaling pathways: a prelude for more. Circ Res. 2010;106(12):1798-806.

37. Gessert $\mathrm{S}, \mathrm{Kuhl} \mathrm{M}$. The multiple phases and faces of wnt signaling during cardiac differentiation and development. Circ Res. 2010;107(2):186-99.

38. Naito AT, Shiojima I, Komuro I. Wnt signaling and aging-related heart disorders. Circ Res. 2010;107(11):1295-303.

39. Lozano-Velasco E, Hernandez-Torres F, Daimi H, Serra SA, Herraiz A, HoveMadsen L, Aranega A, Franco D. Pitx2 impairs calcium handling in a dosedependent manner by modulating Wnt signalling. Cardiovasc Res. 2015;109:55.

40. Minamino T, Kitakaze M, Sato H, Asanuma H, Funaya H, Koretsune $Y$, Hori M. Plasma levels of nitrite/nitrate and platelet cGMP levels are decreased in patients with atrial fibrillation. Arterioscler Thromb Vasc Biol. 1997;17(11): $3191-5$.
41. Tamargo J, Caballero R, Gomez R, Delpon E. Cardiac electrophysiological effects of nitric oxide. Cardiovasc Res. 2010;87(4):593-600,

42. Vandiedonck C, Knight JC. The human major histocompatibility complex as a paradigm in genomics research. Brief Funct Genomic Proteomic. 2009;8(5):379-94.

43. Ter Keurs HE, Shinozaki T, Zhang YM, Wakayama Y, Sugai Y, Kagaya Y, Miura M, Boyden PA, Stuyvers BD, Landesberg A. Sarcomere mechanics in uniform and nonuniform cardiac muscle: a link between pump function and arrhythmias. Ann N Y Acad Sci. 2008;1123:79-95.

44. Akopian AN, Sivilotti L, Wood JN. A tetrodotoxin-resistant voltage-gated sodium channel expressed by sensory neurons. Nature. 1996;379(6562):257-62.

45. Pfeufer A, van Noord C, Marciante KD, Arking DE, Larson MG, Smith AV Tarasov KV, Muller M, Sotoodehnia N, Sinner MF, et al. Genome-wide association study of PR interval. Nat Genet. 2010;42(2):153-9.

\section{Submit your next manuscript to BioMed Central and we will help you at every step:}

- We accept pre-submission inquiries

- Our selector tool helps you to find the most relevant journal

- We provide round the clock customer support

- Convenient online submission

- Thorough peer review

- Inclusion in PubMed and all major indexing services

- Maximum visibility for your research

Submit your manuscript at www.biomedcentral.com/submit
(O) BioMed Central 